\title{
Cognitive Deficits of Patients with Chronic Kidney Disease and Effect of Hemodialysis
}

\section{EGBI Oghenekaro Godwin ${ }^{1 *}$, OGUNRIN Olubunmi ${ }^{2}$ and OVIASU Efosa ${ }^{2}$}

\author{
${ }^{1}$ Department of Internal Medicine, Niger Delta University Teaching Hospital, Okolobiri, Bayelsa \\ State, Nigeria. \\ ${ }^{2}$ Department of Internal Medicine, University of Benin Teaching Hospital, Benin City, Edo \\ State, Nigeria.
}

Background: Cognitive impairment $(\mathrm{Cl})$ has been described in chronic kidney disease (CKD) but the effect of haemodialysis (HD) on cognitive performance of CKD patients

Type: Research

DOI: 10.15580/GJMS.2015.1.012315013

Submitted: $23 / 01 / 2015$

Accepted: 23/01/2015

Published: $30 / 01 / 2015$

*Corresponding Author

Oghenekaro Godwin Egbi

E-mail:drkoge@yahoo.com

\section{Keywords:}

chronic kidney disease, cognitive impairment, endstage renal disease, hemodialysis, iron psychology has not been researched in Sub-Saharan Africa. We studied the cognitive deficits of CKD patients and the short-term effect of HD on cognitive function in end-stage renal disease (ESRD) patients.

Methods: This was a case-controlled analytical study of one hundred and ninety CKD patients and a hundred control subjects recruited and neuro-psychologically assessed with a computer assisted test battery, the Iron Psychology (acronym - FePsy) pre- and post-hemodialysis.

The study was conducted between December 2009 and July 2010 at a tertiary health facility in cosmopolitan southern Nigeria and involved consecutive patients with clinical evidence of CKD based on symptoms and estimated GFR. Demographic, clinical and biochemical variables were related to cognitive performance using Pearson correlation and multiple regression analysis. The outcome measures were scores of psychomotor speed tasks (simple auditory and visual reaction times), recognition memory tests and sustained attention task (binary choice task). The Iron Psychology (FePsy) was used to assess the simple reaction time, complex reaction time, memory and concentration of the CKD patients and controls.

Results: CKD patients performed worse in tasks of information processing speed and memory but compared favorably with controls in tests of concentration. Advancing age, anemia, uncontrolled systolic blood pressure, acidosis and serum chloride derangement were independently associated with cognitive deficits. Acute HD was only associated with improvement in psychomotor speed.

Conclusion: The CKD patients manifested cognitive impairments in memory and psychomotor tasks. Anemia, uncontrolled blood pressure and biochemical derangements predicted cognitive impairment. HD, in the short term, improved psychomotor speed of the patients. 


\section{INTRODUCTION}

End-stage renal disease (ESRD) and less advanced stages of chronic kidney disease (CKD) have been associated with cognitive impairment in several reports. Murray et al (2008), Kurella et al (2008), Hart et al(1983), Murray et al(2006), Kurella et al(2004). Prior studies examining CKD and cognitive function have reported an association of CKD with deterioration in cognitive function, incident dementia and impaired cognitive abilities related to verbal learning, visual attention, mental flexibility and executive functioning. Kurella et al (2004), Kurella (2005a,b) Thornton et al (2007), Halipern et al(2007). The mechanisms proposed as mediators of relations between kidney function and cognition are similar to those that have been advanced to explain relations between other risk factors for cardiovascular disease and cognition including atherosclerosis, clinical stroke, silent stroke, oxidative stress and white matter lesions. Kurella et al(2005a), Seliger and Longstreet Jr (2008).

Patients with CKD have a high prevalence of sub cortical white matter lesions on neuroimaging and a high incidence rate for stroke. Martinez-Vea et al(2006), Fazekas et al(1995). White matter lesions result from subcortical small vessel disease and have been independently associated with severity of kidney disease. Ikram et al (2008). Small vessel cerebrovascular disease leading to the development of subcortical white matter lesions is likely to be accelerated in CKD because these patients often have a clustering of traditional and emerging vascular risk factors, including hypertension, diabetes, hyperlipidemia, elevated oxidative stress and an elevated inflammatory state. Small vessel cerebrovascular disease is the most common cause of vascular dementia with a pattern of cognitive deficits characterized by relatively preserved memory and impairment in domains related to attention, executive function and processing speed. Looi and Sachdev, Roman and Royal, (1999).

In patients with CKD prior to initiating dialysis, cognitive function has been correlated with the severity of renal disease and an increased risk of developing dementia. Kurella et al, 2004, Kurella et al (2008). Dementia prevalence in the hemodialysis population has been reported to be as high as $30 \%$ and is frequently under-recognized. Murray et al (2006), Sehgal et al (1997). The effect of hemodialysis on cognition has remained a subject of controversy. While some studies have reported positive effects of dialysis on at least some cognitive domains. Hart et al(1983), Umans and Pilskin(1998) according to Murray (2008), the dialysis procedure itself may worsen cognitive function. Only few reports on cognitive function of CKD patients exist in developing countries like Nigeria. To the best of the authors' knowledge, the effect of hemodialysis on cognition among Nigerian patients has not been previously studied. This study is the first to assess the pattern of cognitive deficits and the short term effect of hemodialysis on cognitive function in those undergoing hemodialysis.

\section{MATERIALS AND METHODS}

\section{Study population}

All consecutive patients presenting with features of CKD were recruited through the Accident and Emergency (AE) unit, Nephrology clinics, Consultant Outpatient Clinics (COP) and the Dialysis unit of the University of Benin Teaching Hospital (UBTH). UBTH is a 500 bedded tertiary hospital and referral centre providing specialist services in several fields including Nephrology and Neurology.

The study spanned a period of eight months (from December 2009 to July 2010). Two hundred and thirty patients with CKD aged between 16 and 75 years with at least a primary level of education were recruited for the study but only one hundred and ninety satisfied the study criteria. A hundred healthy age and sex matched control subjects with no clinical evidence of CKD or other risk factors for cognitive impairment were recruited from the general population.

Severely dyspnoeic, restless, delirious and uncooperative patients were excluded from the study. In addition, patients on psycho-active agents, those with severely elevated BP $>180 / 110 \mathrm{mmHg}$, upper limb amputees or patients with severe arthritis of the upper limb joints (who consequently may not be able to perform the Fepsy subtests) as well as those with obvious visual or hearing impairments who may not be able to perform the neuropsychological tasks were excluded.

\section{Neuropsychological testing}

The Iron Psychology (acronym - FePsy) test battery was used in assessment of cognitive function. The FePsy is a powerful, automated menu-driven neuropsychological computerized test battery that covers arousal, short term memory, mental speed and vigilance.The Psychological Company (2008). It contains a subset of computerized tests for cognitive neuropsychological functions and a relational database system for storage of the results. It can be administered, stored and printed without extensive knowledge of computers. The FePsy test has been validated for use among Nigerians. Ogunrin et al (2006) and in patients with chronic renal impairment. Elias et al (2009). The FePsy is made of these tasks: binary choice Task, the visual searching task, the Recognition Memory Test (RMT), the finger tapping task, the classification task, the Seashore rhythm task, the computerized visual searching task (CVST), the visual half field tasks and the Corsi Block tapping tasks.

Each of these tasks is better adapted for specific cognitive domains. The RMT can be usefully employed in testing short term memory (STM). The simple reaction time has two versions: auditory and visual. It can test alertness functions as well as speed of activation of the information processing system. The binary choice sensitivity test can also test speed of information processing as well as attention and 
concentration, though it presents a more complex stimulus configuration than the simple reaction time (The Psychological Company (2008).

The signs of cognitive impairment reported in patients with CKD include impaired concentration, alteration in alertness and awareness and loss of recent memory. The RMT, the auditory and visual reaction time as well as the binary choice reaction time were employed in this study to assess memory, psychomotor speed and concentration respectively. With the FePsy, presentation of stimuli and response registration were controlled by a computer. The tests were installed on the hard disks of a Hewlett-Packard (HP) laptop Window XPR with colored monitor. The laptop was kept on a table beside the patient while seated. The screen of the computer was used for stimulus presentation and the keyboard for responses. This allowed for accurate timing, in milliseconds of inter-stimulus interval and responses of the individuals being tested.

Short term memory was assessed using the RMT. The test involves the use of study items which consist of 3 or 4 figures (for the visual memory test) and 4 or 6 words (for the verbal memory test), which are presented simultaneously. The task is divided into 2 phases: a study phase in which the object to be remembered is presented and a test phase in which recognition is tested. A delay of two seconds is automatically allowed between the two phases, the figure or word is presented again during the test phase for recognition. Patients and control subjects with primary level of education were tested using three figures and four words while those with at least secondary level of education were tested using four figures and six words. The results were calculated as percentages of correct responses.

The psychomotor speed was assessed using the simple reaction time. In the visual version, the person being tested reacts as quickly as possible, by pressing a button as soon as a white square in the middle of the computer screen is seen. In the auditory version however, the reaction was to a sound stimulus of $800 \mathrm{Hertz}(\mathrm{HZ})$ generated by the computer. In both versions of the test, the interval between each stimulus was randomly varied by the computer from 2.5 to 4 seconds. The individuals being tested also responded by quickly tapping a button. This was done initially with the dominant hand, and then repeated with the nondominant hand. A total of 30 stimuli each were presented for both hands. The same process was repeated for the control subjects.

In the binary choice reaction task, either a red or a green half-square inch block is displaced in a random sequence in either the left or right half of the screen. The individual being tested was asked to push one of two buttons on either side of the keyboard corresponding to the position of the colored block on the screen. The test is self-paced and continuous which implies that a response is immediately followed by substitution of another block in the same or opposite position. After an initial practice phase of ten stimuli, sixty stimuli were presented. The results showed accuracy and speed (in milliseconds) of responses. Evaluation of results was carried out in the context of complex mental speed, attention and concentration. The sensitivity, specificity and the predictive values of some FePsy subsets among Nigerians and the normative values of FePsy have been documented in the literature. Ogunrin et al (2006).

\section{Clinical evaluation of patients}

Interviewer administered questionnaire was used to obtain socio-demographic data and clinical history. An assessment of chronic kidney disease was made with a history of renal symptoms of greater than or equal to 3 months and an estimated glomerular filtration rate (eGFR) $<60 \mathrm{mls} / \mathrm{min}$. Patients were considered to have ESRD if eGFR $<15 \mathrm{mls} / \mathrm{min}$. Blood pressure was measured with the Accoson Mercury sphygmomanometer. An average of two readings was taken after an interval of 5 minutes. Blood pressure was considered to be well controlled if a value less than $140 / 90 \mathrm{mmHg}$ was obtained and poorly controlled if a higher value was obtained.

The investigations done for the patients at presentation included serum electrolytes, urea, creatinine $(\mathrm{E}, \mathrm{U}, \mathrm{Cr})$ and hematocrit levels. Five milliliters of venous blood was obtained from each subject and dispensed into lithium heparin bottle. Serum creatinine was assayed using modified Jaffe's method while the Urease -Bethelot method was employed for serum urea assay. The flame photometer was used for determination of serum levels of sodium, potassium, bicarbonate and chloride. Electrolyte results were expressed in $\mathrm{mmol} / \mathrm{l}$ while urea and creatinine were measured in $\mathrm{mg} / \mathrm{dl}$. The serum urea and $\mathrm{Cr}$ were repeated an hour post dialysis for all patients that had hemodialysis. Adequacy of dialysis dose was determined by the urea reduction ratio (URR).

URR $=\underline{\text { Pre dialysis serum urea }- \text { Post dialysis serum urea }} \times 100$ Pre- dialysis serum urea

The mean URR was obtained for patients that had 2 sessions of HD by a simple average of the 2 URR values. Patients with eGFR $>15 \mathrm{mls} / \mathrm{min}$, not requiring dialysis as well as the control subjects had their cognition tested once on presentation while those with ESRD had their cognitive function tested just before the commencement of dialysis and repeated an hour after the second session of hemodialysis. Since the first session was an introductory session and lasted for a relatively short period, patients were not tested at this stage.

\section{Ethical consideration}

Ethical approval was obtained from the Ethics and Research Committee of the University of Benin teaching hospital before commencement of the study. Informed consent was obtained from study participants after proper explanation of the procedure.

\section{Data analysis}


Data was analyzed using the Statistical Package for the Social Sciences version 17 (SPSS Inc. Chicago). Results were presented in tabular and graphical forms. Means, median, standard deviation and ranges were calculated for quantitative data. The student's t test was employed to compare the cognitive performances of CKD patients and controls and to compare the pre and post- dialysis performances of ESRD patients. Chi square distribution analysis was used to test for significant differences between proportions and frequencies. One way analysis of variance (ANOVA) was used to compare cognitive performances of patients categorized by stages of CKD. The Pearson correlation was used to test for association of various variables with cognitive performances while multiple linear regression analysis was secondarily used to identify the independent predictors of cognitive impairment. The confidence interval was set at 95\% limit and level of significance was taken as $p<0.05$.

\section{RESULTS}

\section{Characteristics of study population}

One hundred and ninety patients and hundred controls completed the study. The mean ages of the patients were $46.79 \pm 13.47$ and $44.20 \pm 14.71$ years respectively $(z=1.507, p>0.05)$. Majority of the patients (117; $61.6 \%)$ were males. The sex distribution was not statistically different from that of the control group where there were $60(60.0 \%)$ males and $40(40.0 \%)$ females $(p>0.05)$. see Table 1 .

\section{Comparison of cognitive performance of CKD patients and Controls}

The CKD patients had a worse performance than the controls in all cognitive tasks except the binary choice sensitivity task. This was evident from significant differences in the mean scores of the patients and the controls except for the binary choice sensitivity where the difference did not reach statistical significance ( $p>0.05$ ) (see Table 2).

Table 3 shows the mean cognitive scores in the various subtests of FePsy at three different stages of CKD. There was a marked significant difference between these scores as patients with stage 3 CKD had a better score in all subsets of FePsy compared with more advanced stages except in the figure subtest of the memory score where significant statistical difference was not observed $(p>0.05)$. Those with stage 5 CKD had the worst score except for the binary choice sensitivity sub-set where patients with stage 4 CKD had the worst score.

\section{Effect of hemodialysis on cognition}

Twenty six patients had adequate hemodialysis with a mean URR of $66 \%$.Cognitive scores of the CKD patients at baseline and after the second session of hemodialysis are shown in table 4 . The scores were better after hemodialysis. There was significant difference between the pre- and post-dialysis auditory reaction time in both the dominant and non-dominant scores $(p<0.05)$. There was also significant difference in the dominant domain of the visual reaction time preand post- dialysis. Although there was an improvement in the other subsets of

FePsy after dialysis, statistical significance was not reached.

\section{Correlates and predictors of cognitive performance}

The correlates of cognitive performance in the CKD patients were age, systolic and diastolic blood pressure, haematocrit level, serum creatinine, serum urea, serum creatinine, serum chloride but this association differed across various cognitive domains.(see Table 5).

Older age was a predictor of poor performance in the auditory reaction times $(p=0.005$ and $p=0.04$ for auditory dominant and non-dominant reaction times respectively), visual reaction times $(p=0.001$, and $p<0.001$ for dominant and non-dominant reaction times respectively) and the binary choice reaction times $(p<0.001)$.Similarly, higher systolic blood pressure predicted both auditory reaction times $(p<0.001)$, the visual dominant reaction time $(p<0.001)$ the binary choice reaction time $(p=0.03)$ as well as both word subtest of memory scores $(p=0.01)$ and figure subtest of memory scores $(p=0.001)$. Lower serum bicarbonate was a predictor of poor performance in both auditory reaction times $(p<0.001$ and $p=0.02)$ for the auditory dominant and non-dominant reaction times respectively) and the visual dominant reaction times $(p=0.001)$ while low haematocrit was an independent determinant of poor performance in the auditory $(p=0.006)$ and visual non-dominant reaction domains $(p=0.02)$ as well as the binary choice reaction domain $(p=0.008)$ and the word score $(p=0.03)$. Poor performance in the auditory non-dominant reaction time was also predicted by higher level of serum urea $(p<0.001)$ while higher serum chloride determined poor performance in the binary choice sensitivity $(p=0.01)$ and the word score domains $(p=0.03)$.

\section{DISCUSSION}

\section{Comparison of cognitive performance}

The CKD patients performed significantly worse than controls in all cognitive tasks except the binary choice sensitivity where there was no significant difference. Thus, patients with CKD may be at higher risk of memory impairment and may have general slowing of psychomotor speed and thought processes. However, their attention and concentration abilities may not differ from the general population. This finding corroborates an earlier report by Ogunrin et al (2006). However, the reaction times for our control subjects were higher than the normative values reported by Ogunrin and Adamolekun (2007) in another study. This may be due to differing methodology. A desktop with separate keyboard resting on the person's laps was used in the 
normative study while in this study a laptop resting on the table was used. In the latter case, the reaction times are expected to be more prolonged due to the 'hand - reaching effect.' This may also explain the disparity in the word scores between the control subjects in this study and the normative subjects.

Selective impairment in cognitive impairment among CKD patients was also reported by Elias et al (2009), who demonstrated a decreased performance level within specific domains of cognition in association with modestly lower renal function. They reported lowered ability in attention, concentration, visual processing speed, psychomotor performance and planning resulting in difficulty with completing an organized visual search in their CKD patients. They also observed deficits in visual pattern recognition, immediate and delayed memory for visual materials, and organization and problem solving in a visualspatial context. In contrast, neither working memory nor verbal episodic memory was significantly associated with CKD. In our study however, verbal memory deficit was seen in the CKD subjects. This may not be surprising as our population consisted of moderate and advanced CKD patients while Elias' population included those with relatively mild degrees of renal impairment. Studies have shown that more severe cognitive impairment extending to memory usually occur in the context of more advanced CKD. Murray (2008), Griva et al(2006).

According to Pugh and Lipsitz (2002), impairments in the domains of attention and processing speed, executive function, and language with a relative preservation of memory is reminiscent of the cognitive changes associated with the early manifestations of vascular dementia secondary to small vessel cerebrovascular disease. This pattern of cognitive deficit has been associated with manifestations of cerebral small vessel disease such as white matter lesions. Ylikoski et al (1993), DeGroot et al (2000).Subcortical white matter lesions are thought to be highly prevalent in advanced CKD and associated with renal disease severity. Ikram et al (2008). Our study similarly showed a decline in cognitive performance with increased renal severity. There was also a graded relationship between stages of CKD and cognitive performance for most domains of FePsy. Those with higher stages had progressively worse performance in the various subsets of FePsy except the figure score subset of the RMT, implying that mental speed, attention and concentration as well as the verbal memory may worsen as renal function declines. The non- verbal memory may however not change.

\section{Predictors of cognitive performance}

Older CKD patients were more likely to have impairment in psychomotor functions and complex mental speed compared with younger ones. This finding is consistent with available literature even in those without CKD. Ogunniyi et al (2007).

Psychomotor speed was predicted by age, systolic BP, hematocrit, serum urea and creatinine as well as bicarbonate levels since these were independent predictors of the reaction times. Lower hematocrit, higher serum urea and creatinine as well as lower serum bicarbonate were all associated with poor cognitive performance.

Anemia has been associated with cognitive impairment in patients with chronic renal impairment as well as in those without CKD. Atti et al (2006). Serum creatinine was found to be the most potent variable affecting cognitive performance in a Nigerian study. Elias et al (2009). Although acidosis may not have been directly linked with cognitive decline in CKD patients in the existing literature, lactic acidosis has been related to neurological impairment in patients with mitochondrial mypoathy, encephalopathy, and stroke episodes (MELAS), thus suggesting a possible role of acidosis in cognitive decline. Kaufman et al (2004).

According to Goldman et al (1989), acidotic environments in vitro have also been shown to be toxic to neurons and glia cells. Systolic blood pressure was negatively related to mental speed as well as both verbal and visual memory while diastolic blood pressure was negatively associated with the mental speed of the patients but not with memory. This finding is similar to that of Kuo et al (2004) who reported an association between blood pressure elevation and selective impairment in the executive function of the brain. Elias et al (2004) also found selective impairment in the visualization subset in both young and elderly people with elevated blood pressure.

Age, systolic blood pressure and hematocrit were independent determinants of the complex mental speed of these patients with older, hypertensive patients having low hematocrit performing worse. Attention and concentration were predicted by the serum chloride levels of these subjects with those with higher serum chloride levels, performing worse. Alterations in the level of serum chloride have similarly been linked to disturbed thought processes in some literature. Smelter et al (2009).

Higher systolic blood pressures and low haematocrit were positively associated with poor performance in verbal memory. Poor performance in visual memory was similarly associated with high systolic blood pressures and low serum bicarbonate. Systolic blood pressure was negatively related to mental speed as well as both verbal and visual memory while diastolic blood pressure was negatively associated with the mental speed of the patients but not with memory.

\section{Effect of haemodialysis on cognitive performance}

The study also demonstrated an improvement of cognitive performance after haemodialysis from baseline in all the FePsy subtests. However, only those of the auditory reaction and the visual (dominant) tasks reached statistical significance. Thus acute hemodialysis may improve psychomotor speed of CKD patients but may have no significant effect on verbal or visual memory, concentration or complex mental speed. Unfortunately, there is paucity of data 
for adequate comparison. Umans and Pilskin (1998) however reported that adequate dialysis could mitigate the effects of uremic toxins on the brain, and thus lead to an improvement I n cognitive function.

They opined that well dialyzed patients were unlikely to manifest significant uremic neurocognitive deficits especially in spheres related to sustained attention or mental processing speed.

In a report by Hart et al (1983), non-dialyzed azotaemic patients were impaired on several tasks relative to dialysis patients. Thimmaiah et al (2012) reported improvement in neuropsychological measures of memory and attention 24 hours after hemodialysis. There was however no improvement in tests of attention following dialysis in our study. Differing methodology may account for this disparity. Our study population was made up of both moderate and advanced CKD while all the patients in Thimmaiah's study had advanced renal failure requiring dialysis. Jassal et al. reported selective improvement in cognition (attention, working memory, psychomotor efficiency and information processing speed) with increasing frequency of dialysis. Jassal et al (2006). However, learning efficiency did not improve. On the other hand; task performance was observed to be consistently worse during haemodialysis than before commencement of the session in a report by Smith et al.37 This may have been due to temporary imbalance in brain fluid and electrolyte imbalance as the assessment was carried out during the dialysis procedure. In our study, cognitive test was done immediately after, not during the procedure. It is therefore possible that though there may be some transient cognitive deficits occurring during the haemodialytic session, an overall improvement in some cognitive domains after the session may occur.

\section{CONCLUSION}

This study showed that CKD patients manifest cognitive deficits in memory, psychomotor speed and thought processes which may be more obvious with increasing severity of renal disease.

Anemia, uncontrolled blood pressure and biochemical derangements may contribute to these cognitive deficits. The study also revealed that acute haemodialysis may be beneficial in improving some of these cognitive functions especially. psychomotor speed and thought processes. Initiating dialysis in CKD patients may therefore help in improvement of some aspects of cognitive function at least in the short term, though may not affect patient's memory, attention or concentration.

To the best of our knowledge, this study is the first of its kind in Nigeria to directly assess the effect of haemodialysis on cognition in patients with renal disease. Another major strength of this study lies in the use of a computerized, previously validated test instrument in the evaluation of cognitive abilities which also attempts to identify the specific cognitive domain affected. Small sample size of patients who had HD and non-inclusion of patients with no formal education and those who were severely may limit the extent of generalisability of our findings. However, this does not invalidate our findings. Further studies need to be carried out on a larger scale to establish determinants of cognitive impairment in CKD patients and how it can be ameliorated as well as to further elucidate the role of dialysis in cognition on a short and long term basis.

\section{REFERENCES}

Atti AR, Palmer K, Volpato S, Zuliani G, Winblad B, Fratiglioni $L$ (2006). Anaemia increases the risk of dementia in cognitively impaired intact elderly. Neurobiol Aging; 2: 278-284.

De Groot JC, de Leeuw FE, Oudkerk M et al. (2000). Cerebral white matter lesions and cognitive function: The Rotterdam Scan Study. Ann Neurol.; 47:145-151.

Elias MF, Elias PK, SeligerSL (2009). Chronic kidney disease, creatinine and cognitive functioning. Nephrol Dial Transplant.; 24 (8): 2446-2452.

Elias PK, Elias MF, Robbins MA, Budge MM (2004). High blood pressure related decline in cognitive function. Hypertension; 44:631-636.

Fazekas G, Fazekas F, Schmidt R et al.(1995). Brain MRI findings and cognitive impairment in patients undergoing chronic hemodialysis treatment. $J$ Neurol Sci;134:83-88.

Griva K, Thompson D, Jayasena D et al (2006) Cognitive functioning pre- to post-kidney

transplantation-a prospective study. Nephrol Dial Transplant, 21:3275-3282

Hailpern SM, Melamed ML, Cohen HW, et al.(2007). Moderate chronic kidney disease and cognitive function in adults 20 to 59 years of age: Third National Health and Nutrition Examination Survey (NHANES III). J Am Soc Nephrol; 18:22052213.10.

Goldman SA, Pulsinelli WA, Clarke WY, Kraig RP, Plum F. The effect of extracellular acidosis on neurons and glia in vitro. J Cereb Blood Flow Metab 1989; 9(4):471-477.

Hart RP, Pederson JA, Czerwinski AW et al (1983). Chronic renal failure, dialysis, and neuropsychological function. J Clin Neuropsychol; 5:301-312.

Ikram MA, Vernooij MW, Hofman A et al (2008). Kidney function is related to cerebral small vessel disease. Stroke; 39:55-61.

Kaufman P, Shungu DC, Sano MC et al. Kaufman P, Shungu DC, Sano MC et al. (2004). Cerebral lactic acidosis correlates with neurological impairment in MELAS. Neurology; 62:1297-1302.

Kurella M, Chertow GM, Luan J, et al. (2004). Cognitive impairment in chronic kidney disease. J Am Geriatr Soc; 52:1863-1869.

Kurella M, Chertow GM, Fried LF, et al.( 2005). Chronic kidney disease and cognitive impairment in the elderly: the health, aging and body composition study. J Am Soc Nephrol; 16:21272133.

Kurella M, Yaffe K, Shlipak MG et al. (2005); Chronic kidney disease and cognitive impairment in menopausal women. Am J Kidney Dis; 45:66-76. 
Kuo HK, Sorond F, lloputaife I, Gagnon M. (2004). Effect of blood pressure on cognitive function of elderly persons. J Gerontol A Biol Sci Med Sci; 59(11):1191-1194.33.

Kurella M, Yaffe K, Shlipak MG, et al.( 2005). Chronic kidney disease and cognitive impairment in menopausal women. Am J Kidney Dis 2005; 45:66-76.

Kurella TM, Wadley V, Yaffe MD et al. Kidney function and cognitive impairment in US adults: the Reasons for Geographic and Racial Differences in Stroke (REGARDS) Study. Am J Kidney Dis; 52:227-234.

Looi JC, Sachdev PS (1999). Differentiation of vascular dementia from $A D$ on neuropsychological tests. Neurology; 53:670-678.

Madero M, Gul A, Sarnak MJ (2008). Cognitive function in chronic kidney disease. Semin Dial 21:29-37.

Martinez-Vea A, Salvado E, Bardaji A et al.(2006). Silent cerebral white matter lesions and their relationship with vascular risk factors in middleaged pre-dialysis patients with CKD. Am J Kidney Dis; 47:241-250.

Murray AM. (2008). Cognitive impairment in the Ageing dialysis and chronic kidney disease population: An occult burden. $A m \quad J \quad K i d$ Dis;15(2):123-132.

Murray AM, Tupper DE, Knopman DS, et al.( 2006). Cognitive impairment in hemodialysis patients is common. Neurology, 67:216-223.

Murray AM, Pederson SI, Tupper DE et al (2007). Acute variation in cognitive function in hemodialysis patients. A cohort, with repeated measures. Am J Kidney Dis; 50:270-278.

Ogunniyi A, Hall KS, Gureje O. et al ( 2007). Cognitive decline and its determinants in a sample of elderly Yoruba. Proceedings of the Silver Congress of the International Psychogeriatric Association. Oct 14-
18, Osaka, Japan. In: International Psychogeriatrics 19(S1):45-46.

Ogunrin OA, Adamolekun B. (2007). Cognitive Neuroassessment in Nigerian Africans- predictive validity of a computerized testing. Ann Biomed Sci; $6(1): 28-44$.

Ogunrin OA, Unuigbe IE.Azubike C (2006). Memory and perceptuo -motor performance in Nigerians with chronic renal impairment. Med Sci Monitor, 12(12): 535-539.

Pugh KG, Lipsitz LA. The microvascular frontalsubcortical syndrome of aging. Neurobiol Aging 2002; 23:421-431.

Roman GC, Royall DR(1999). Executive control function: a rational basis for the diagnosis of vascular dementia. Alzheimer Dis Assoc Disord.; 13(Suppl 3):S69-S80.

Sehgal AR, Grey SF, DeOreo PB, Whitehouse PJ (1997). Prevalence, recognition, and implications of mental impairment among hemodialysis patients. Am J Kidney Dis. 1997; 30:41-49.

Seliger SL, Longstreth WT Jr. (2008). Lessons about brain vascular disease from another pulsating organ, the kidney. Stroke; 39:5-6.

The psychological company. FePsy, a neuropsychological computerized test battery (2008) [cited 2009 Oct 08]; Available from: URL: http://www.fepsy.com.

Thornton WL, Shapiro RJ, Deria S, et al. 2007 Differential impact of age on verbal memory and executive functioning in chronic kidney disease. $J$ Int Neuropsychol Soc; 13:344-353.

Umans JG, Pilskin NH (1998). Attention and mental processing speed in hemodialysis patients. $A m \mathrm{~J}$ Kidney Dis 32(5):749-751.

Ylikoski R, Ylikoski A, Erkinjuntti T, Sulkava R, Raininko R, Tilvis $R(1993)$. White matter changes in healthy elderly persons correlate with attention and speed of mental processing. Arch Neurol 50:818-824. 